\title{
Utilización de marcadores bioquímicos como método no invasivo en la determinación de la viabilidad embrionaria en Fertilización In-Vitro
}

\section{Biochemical markers used as a non-invasive method for embryos viability determination in In-vitro Fertilization}

Pág. 18-19

Jannin Alfaro, Pamela Bustamante, Alex Duarte, Priscila Fernández, Sofía Núñez, Jeannethe Zúñiga1.

Estudiantes de Microbiología Universidad de Ciencias Médicas (UCIMED), San José, Costa Rica.

1 Directora de Nutrición, Universidad de Ciencias Médicas (UCIMED), San José, Costa Rica.

\section{Resumen}

Actualmente se ha optado por la implementación de la metabolómica en la fertilización in vitro como un método no invasivo para determinar la viabilidad de los embriones, utilizando la medición de productos secretados o consumidos por el embrión en su medio de cultivo. Investigaciones realizadas han comprobado que moléculas como los carbohidratos, aminoácidos, entre otras, se pueden utilizar como marcadores bioquímicos, ya que se han relacionado con un buen desarrollo del embrión.

Palabras claves: marcador bioquímico, fertilización in vitro, metabolómica, embrión

\section{Abstract}

Nowadays the metabolomics has been implemented in the In Vitro Fertilization as a noninvasive method for embryo viability determination, using the measurement of secreted and consumed products in the culture. Several investigations have proven that molecules as carbohydrates, amino acids, among others, can be used as biochemical markers because they have been related with an excellent embryo development.

Key Words: biochemical markers, In Vitro Fertilization, metabolomics, embryo

\section{Introducción}

Durante el proceso de fertilización In Vitro se realizan pruebas para determinar la viabilidad de los embriones, ya que su selección optimiza las posibilidades de embarazo y se disminuyen los embarazos múltiples y sus complicaciones
(Sallam et al, 2016). Se hacen mediante métodos invasivos y no invasivos, un ejemplo de estos últimos es la determinación de marcadores bioquímicos o metabolómica (Calomarde et al, 2016).

\section{Metabolómica}

Detecta y cuantifica los metabolitos tanto endógenos y exógenos (Yanes, s.f.). El análisis del metaboloma embrionario correlaciona la actividad metabólica con la viabilidad embrionaria y su capacidad de implantación.

\section{Medio de cultivo utilizado para viabilidad del embrión}

Actualmente se cuentan con dos tipos de medio para el cultivo del embrión y mantener su viabilidad: sistemas de cultivo secuencial y sistemas de monocultivo. Apoyan el desarrollo del cigoto en la etapa de blastocisto y son formulados para las etapas de fertilización, escisión y blastocisto. Un aditivo importante es la albúmina sérica humana, la cual genera las condiciones adecuadas para el embrión (Montskó, 2017).

\section{Marcadores bioquímicos en el medio de cultivo}

Existen distintos productos en los medios de cultivo que son consumidos o secretados por el embrión, por lo que es de suma importancia evaluarlos (Montskó, 2017), ya que determinarán la viabilidad embrionaria. Entre ellos se encuentran: evaluación del metaboloma del embrión, medición del fragmentos s-HLA-G, BBHCG, fragmento haptoglobina $\alpha-1$, leptina, ubiquitina, y concentración del factor activador de plaquetas. (Sallam et al, 2016).

Entre los más importantes se encuentran el 
metabolismo de carbohidratos y el turnover de aminoácidos, donde el alto consumo de piruvato y glucosa, así como el consumo de leucina y secreción de alalina, se han relacionado con mejor viabilidad del embrión y desarrollo de blastocitos de mejor calidad respectivamente. El fragmento soluble del HLA-G tiene importancia a nivel de la implantación del embrión y tolerancia del feto por parte de la madre (Sallam et al, 2016) (Lundin \& Ahlstrom, 2015).

\section{Métodos para la determinación de marcadores bioquímicos}

Se utiliza cromatografía líquida de alta eficacia (HPLC) para la determinación del turn over en aminoácidos y espectroscopía de infrarrojo cercano (NIR) que permite establecer perfiles metabolómicos para cada embrión (Vergouw et al, 2008), también mediante espectrometría de masas (Kovács et al, 2016), ELISA, el cual permite la determinación de antígenos como HLA-G (Lundin \& Ahlstrom, 2015), analizadores de termoquimioluminiscencia y electroquímica para la medición del estrés oxidativo (Sallam et al, 2016).

\section{Ventajas y desventajas de la utilización de marcadores bioquímicos}

La determinación del metaboloma presenta resultados confiables, estandarizados y requiere poca muestra (Lundin \& Ahlstrom, 2015). No obstante, la medición de metabolitos puede resultar dificultoso por su complejidad química y heterogeneidad, por limitaciones intrínsecas de cada técnica y la falta de estandarización de los métodos (Lundin \& Ahlstrom, 2015).

\section{Conclusiones}

Al determinar la viabilidad embrionaria mediante los componentes del medio de cultivo que son consumidos o secretados por el embrión, permite disminuir el número de estos que van a ser implantados, y por ende se evita un embarazo múltiple.
Aún no se puede afirmar que, la utilización de marcadores bioquímicos es una técnica exitosa en todas sus ocasiones, por lo que se necesitan de múltiples estudios adicionales para llegar a conocer más acerca de este tema.

\section{REFERENCIASBIBLIOGRAFICAS:}

Calomarde, C., Carmona, L., Guerra, I., Gutiérrez, D., Santana, A. \& Domingo del Pozo, J. (2016). Técnicas invasivas y no invasivas en el diagnóstico de embriones humanos. Rev. Iberoam. Fert Rep Hum, 33; 67-72. Obtenido de:http://www.revistafertilidad.org/rif-articulos/t-e acutecnicas-invasivas-y-no-invasivas-en-el- diagn-oacute-sticode-embriones-humanos/2 43

Kovács, G., Montskó, G., Zrínyi, Z., Farkas, N., Várnagy, Á. \& Bódis, J. (2016). Non-Invasive Assessment of Viability in Human Embryos Fertilized in Vitro. EJIFCC, 27(2), 112-121. Obtenido de: https://www.ncbi.nlm.nih.gov/pmc/articles/PM C4975227I

Lundin, K., Ahlstrom, A. (2015). Quality control and standardization of embryo morphology scoring and viability markers. Reproductive BioMedicine Online, 31(4), 459-471.Obtenido de: https://www.sciencedirect.com/ science/article/pii/S1472648315003624

Montskó, G., Zrínyi, Z., Várnagy, A., Bódis, J., Kovács, G. (2017). Non-Invasive Assessment of the Embryo Viability via the Analysis of the Culture Media. En Bin WuEd.) Embryo Cleavage. IntechOpen. Obtenidode:https://www. intechopen.com/books/embryo-c leavage/non-invasiveassessment-of-the-em bryo-viability-via-the-analysis-of-theculture- media

Sallam, H. N., Sallam, N. H., \& Sallam, S. H. (2016). Noninvasive methods for embryo selection. Facts, views \& vision in ObGyn, 8(2), 87-100. Obtenido de: https://www.ncbi.nlm. nih.gov/pmclarticles/PM C5130307/pdf/FWinObGyn-8-87. pdf

Vergouw, C., et al. (2008). Metabolomic profiling by nearinfrared spectroscopy as a tool to assess embryo viability: a novel, non-invasive method for embryo selection. Human Reproduction, 23 (7); 1499-1504. https://doi.org/10.1093/ humrep/den111

Yanes, O. (Sin fecha). Metabolómica: la ciencia ómica más multidisciplinaria. Sociedad Española de Bioquímica y Biología Molecular. Obtenido de: https://www.sebbm. es/revista/articulo.php?id=42\&url=metabolomica-la cienciaomica-mas-multidisciplinaria

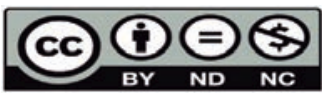

